\title{
THE EFFECT OF THREE ROOT CANAL SEALERS TO ROOT CANAL DENTIN WALLS
}

\author{
Eid B. Mohamed*, Elsayed.R.Hassan** and Kataia M. Medhat**
}

\begin{abstract}
Aim: To compare push-out bond strength of gutta percha and three root canal sealers AH-Plus, MTA Fillapex, Gutta flow 2.

Materials \& Methods: Sixty human freshly extracted lower premolars were prepared using nickel titanium Revo-S files till AS 40 finishing file and divided into three equal groups $n=20$, Group I: Gutta percha/AH-Plus sealer, Group II: Gutta percha/ MTA Fillapex, Group III: Gutta percha/Gutta flow 2. Each root was sectioned in a horizontal plane, from each root three sections with $2 \mathrm{~mm}$ in thickness were obtained at 1, 4, $9 \mathrm{~mm}$ from apex representing apical, middle, and coronal respectively. The push-out bond strength was determined for each root slice using universal testing machine. Data were analyzed using one-way ANOVA test.
\end{abstract}

Results: AH-Plus showed the highest bond strength while the Gutta flow 2 showed the lowest, there was no statistically significant difference between the three levels apical, middle \& coronal.

Conclusions: The composition of endodontic sealers affects their push out bond strength, where the higher push out bond strength was found in GP/AH Plus group and the lowest in gutta flow 2 , while there was insignificant difference in adhesion within the tested groups at different levels.

\section{INTRODUCTION}

Sealing ability of root canal filling is of crucial significance for the long-term success of root canal treatment. The Washington study of endodontic success and failures has attributed nearly $60 \%$ of endodontic failures to incomplete obturation of $\operatorname{root}^{1}$

While Gutta-percha has generally been recognized as the gold standard root canal filling materials however, it does not bond to the internal tooth structure resulting in the absence of complete seal.
Researchers are evolving to provide endodontic sealer that has the ideal physical and biological properties. In the last decades a wide variety of endodontic sealers were commercially available and clinically used, classified according to chemical components into: zinc-oxide-eugenol sealers, Sealers containing calcium hydroxide, and resinbased, glass-ionomer-based, silicone-based, and bioceramic sealers ${ }^{2}$.

\footnotetext{
* Lecturer, Endodontic Department, Faculty of Dentistry, Ahram Canadian University.

** Lecturer, Endodontic Department, Faculty of Dentistry, Almenia University.
} 
While none of the currently available sealers owns all the ideal properties, however some sealers posses more advantages than others have. Disadvantages of the current sealers are being used such as, dimensional changes of root canal sealers over time that may cause gaps along the sealer/ dentin or sealer/gutta-percha interface, discoloration of the tooth structure and dissolution over time, leaving unacceptable voids. Countless attempts have been made to resolve these problem, hence the innovations of endodontic obturation techniques, materials and sealers.

$\mathrm{AH}$ Plus is an epoxy-resin based sealer that proved to have low solubility and disintegration and good adhesion ${ }^{3}$ Gutta flow 2 is the first sealer/ gutta-percha combination which is flowable at room temperature that can be used as sealer as well as obturating paste without a solid master cone ${ }^{4}$. It consists of a polydimethylsiloxane matrix, which is highly filled with very finely ground gutta-percha and nano silver. The sealer part of Guttaflow is highly thixotropic, and with its fine grain size $(<9$ $\mu \mathrm{m})$, the material flows well under slight pressure into the finest lateral canals ${ }^{5}$.The manufacturer claims a better seal and good adaptability because of good flowability and the fact that this material expands slightly $(0.2 \%)$ on setting, enhancing its adaptation to root dentin walls ${ }^{6}$

A new calcium silicate-based sealer (MTA

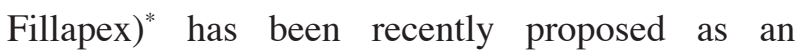
endodontic filling material. The strong interest in developing mineral trioxide aggregate (MTA)-based endodontic materials is because of the excellent biocompatibility, bioactivity, and osteoconductivity of $\mathrm{MTA}^{7}$

\footnotetext{
* Angelus, Londrina, Parana.

** micro mega Besancon, Cedex, France.

*** Dentsply DeTrey GmbH, Konstanz, Germany.

**** Angelus, Londrina, Brazil.

***** Colten/Whaledent GmbH, Langenau Germany.
}

MTA Fillapex is a sealer that is composed of MTA, salicylate resin, natural resin, bismuth oxide, and silica. A recent study showed that this sealer has suitable physico- chemical properties, such as good radiopacity, flow, and alkaline $\mathrm{pH}^{8}$ The manufacturer states that it has a great working time, low solubility, and easy handling This study was conducted to compare the bond strength of three different root canal sealers to root canal dentin.

\section{MATERIALS AND METHODS}

Sixty human freshly extracted lower premolars with fully developed roots, single, straight canal were selected for this study. All teeth were cleaned from calculus and any soft tissues by using ultrasonic scaler.

All the teeth were decoronated using a water coolant low speed diamond disc to standardize all the root lengths to $15 \mathrm{~mm}$. Patency of the root canal was established using k-file size 10. All the root canals were prepared using nickel titanium Revo-S $\mathrm{S}^{* *}$ files till AS 40 finishing file. During the preparation $3 \mathrm{ml}$ of $\mathrm{NaOCl} 5.25 \%$ were used between each file, the prepared root canals were rinsed with $1 \mathrm{~mL}$ of $17 \%$ Ethylenediamine Tetraacetic Acid (EDTA) followed by a final rinse with $5 \mathrm{~mL}$ distilled water, and were dried with paper points. All of the sixty teeth were randomly divided into one of three groups according to the root canal sealer used.

Group I: Root canals were obturated using gutta percha AS 40 \& AH-Plus sealer****

Group II: Root canals were obturated using gutta percha AS 40 \& mineral trioxide aggregate (MTA)-based root canal sealer (MTA Fillapex ${ }^{* * * *}$ )

Group III: Root canals were obturated using gutta percha AS $40 \&$ Gutta flow $2^{* * * * *}$. 
All roots were stored at $37^{\circ} \mathrm{C}$ and $100 \%$ humidity for 1 week to allow complete setting of the root canal sealers.

\section{Push-out bond strength test}

Each root was sectioned in a horizontal plane perpendicular on the long axis using a water-cooled precision saw*. From each root three sections with $2 \mathrm{~mm}$ in thickness were obtained at 1, 4,9 $\mathrm{mm}$ from apex representing apical, middle, and coronal respectively, each specimen was measured using digital caliper (fig 1). Oval root sections were discarded and replaced with new circular specimens.

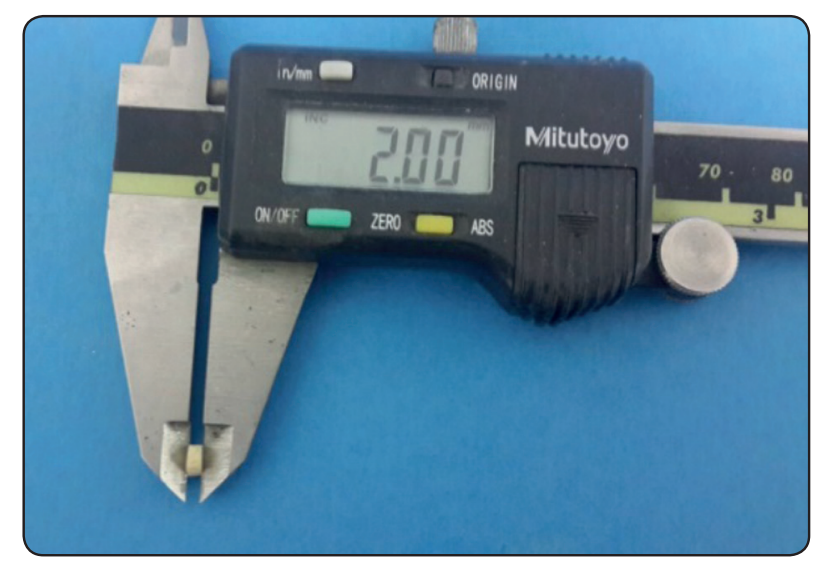

Fig (1) Showing $2 \mathrm{~mm}$ thickness recorded using digital caliper

From each group, each slice was marked on its coronal side with an indelible marker to ensure that the direction of the plunger push was in the apical to coronal direction. Each root slice was mounted in custom made loading fixture metallic block with circular cavity at the middle, this cavity for specimen housing having a central whole to facilitate displacement of extruded filling material, then subjected to compressive loading at a crosshead speed of $0.5 \mathrm{~mm} / \mathrm{min}$ via a computer controlled materials testing machine** (fig 2). The plunger tip was sized and positioned to touch only the filling, without stressing the surrounding dentin.

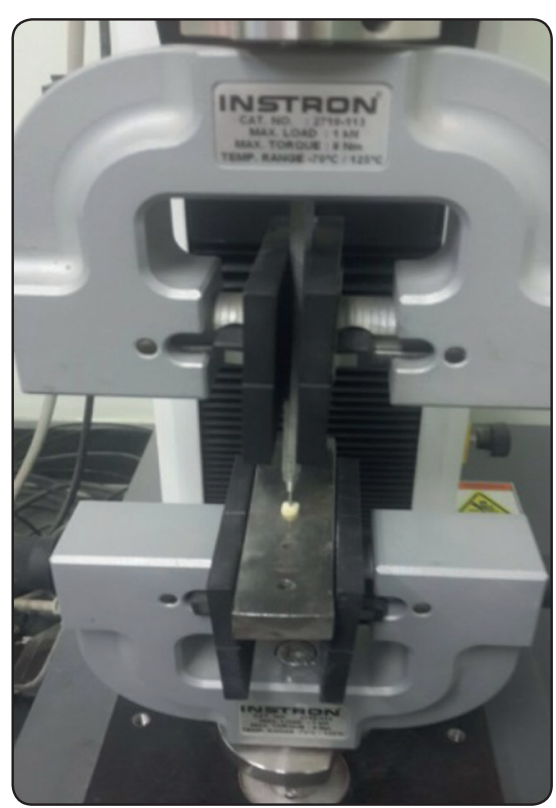

Fig. (2) Showing root slice mounted in universal testing machine

Failure manifested by extrusion of filling piece and confirmed by sudden drop along load-deflection curve recorded by Bluehill 3 computer software.

The bond strength was expressed in MPa by dividing the load in Newtons $(\mathrm{N})$ by the area of the bonded interface $\left(\mathrm{mm}^{2}\right)$. The area was calculated according to the following formula.

\section{$[A=(3.14 \times$ r1X 3.14x r2) L],}

rl apical radius,

$r 2$ coronal one,

$L=[(\mathrm{r} 1-\mathrm{r} 2) 2+\mathrm{h} 2] 0.5$

$h$ is the thickness of the sample in millimeters.

The push-out bond strength was determined for each root slice. Values were recorded, tabulated for each group and statistically analyzed.

\footnotetext{
* Isomet 4000, Buehler, USA.
}

** Instron, England. 


\section{RESULTS}

The data in Table (1) shows the means, standard deviation (SD) of push-out bond strength values represented by $\mathrm{MPa}$, of different tested root segments filled with different root canal sealers. The results also were plotted in a bar chart (fig 3).

In the coronal segment, there was significant difference between the mean push-out bond strength values of all the tested sealers. The AH Plus group showed mean value of $(\mathbf{6 . 2 3} \mathbf{\pm 1 . 5 6})$ which was the highest push out bond strength followed by the Fillapex group and Guttaflow.

Concerning the middle segments, there was significant difference between all tested materials where The AH Plus group showed highest push out bond strength value of $(7.32 \pm .1 .76)$ followed by the Fillapex group and Guttaflow respectively $(3.73 \pm .98)$ and $(1.80 \pm 1.34)$.

The AH Plus group showed significantly higher push out bond strength value of $(6.97 \pm 2.14)$ for the apical segment, followed by Fillapex group (2.56 $\mathbf{\pm 0 . 6 5 )}$, while Guttaflow showed the lowest mean values of $(\mathbf{1 . 7 3} \pm \mathbf{0 . 5 3})$.

Comparing the results of the root segments of each tested material there was insignificant difference between the mean push out bond strength values of the apical, middle and coronal segments in all tested sealers.

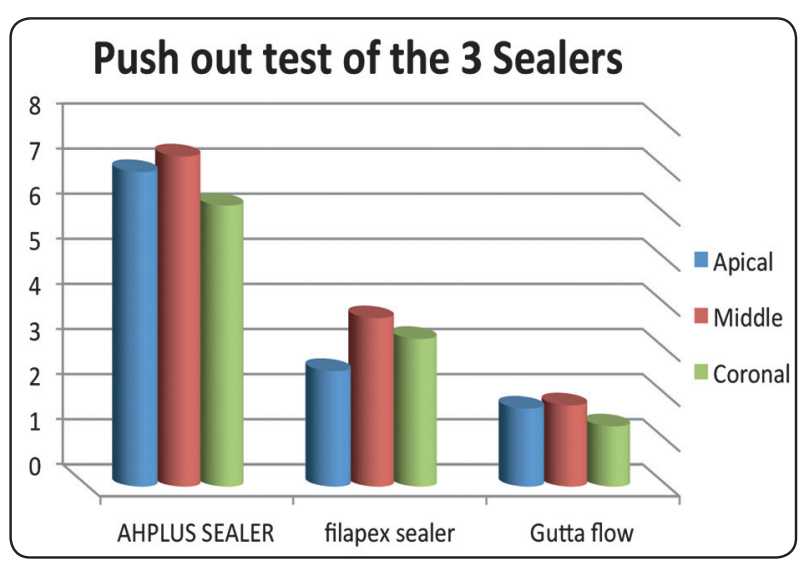

Fig. (3) Comparison of the three tested sealers at three levels

\section{DISCUSSION}

Root canal seal and filling integrity is an important component for the success of endodontic therapy. Bond strength of the endodontic sealers directly affects the sealing ability of our filling material and resists its displacement, statically, during function and even through the process of post preparation ${ }^{9,10,11}$

That is why it was of high importance to shed a light on bond strength ability of different sealers in this study.

Bond strength was tested through push out test as it has become widely accepted in terms of recording, reproducibility and simplicity, in addition the strength of attachment between radicular dentine

TABLE (1) The means, standard deviation (SD) values and results of ANOVA test for the comparison between push out bond strength of the tested materials

\begin{tabular}{|c|c|c|c|c|c|c|c|c|}
\hline Site & \multicolumn{2}{|c|}{ Apical } & \multicolumn{2}{|c|}{ Middle } & \multicolumn{2}{|c|}{ Coronal } & \multirow{2}{*}{ Sig. } & \multirow{2}{*}{ F-value } \\
\hline Sealers & Mean & St. dev & Mean & St. dev & Mean & St. dev & & \\
\hline AHPLUS SEALER & 6.97 (a) & 2.14 & 7.32(a) & 1.76 & $6.23(a)$ & 1.56 & 0.53 & 0.644 \\
\hline Fillapex sealer & $2.56(b)$ & .65 & $3.73(b)$ & .98 & $3.27(b)$ & 1.50 & 0.163 & 2.011 \\
\hline Gutta flow & $1.73(\mathrm{c})$ & .53 & $1.80(\mathrm{c})$ & .67 & $1.34(\mathrm{c})$ & .50 & 0.283 & 1.353 \\
\hline F-value & \multicolumn{2}{|c|}{31.61} & \multicolumn{2}{|c|}{36.391} & \multicolumn{2}{|c|}{25.812} & & \\
\hline
\end{tabular}

Means with the same letter are not significant, while different letter indicate statistical significant difference. 
and sealer material can easily be determined either in high or low levels ${ }^{12}$.

Micro tensile bond strength test was not used due to its inferior homogeneity in stress distribution. In addition to the necessity to measure multiple slices in this study which is only applicable in the push out bond strength test ${ }^{13,14}$.

AH plus is considered a bench mark gold standard due to its excellent properties such as low solubility, small expansion, adhesion and high sealing ability ${ }^{15}$. The second sealer used in this study to be compared was the MTA filapex sealer, which was used to its ability to form hydroxyl appetite crystals in the apical and middle parts of the canal. MTA sealers are characterized by being highly biocompatible to periodontal tissues ${ }^{16}$.

The third sealer compared in this study was the guttaflow filling material, which is a modification of Roekoseal auto mix, and contains Gutta-percha in powder form with particles less than 30 micrometers. The manufacturer claims it has high adaptability and sealing properties due to its inherent flow and post set expansion ${ }^{4}$.

The tests were done on extracted teeth to try and mimic the clinical situation and to come in accordance with the international consensus that natural teeth are the most frequent subjects used in vitro studies with uniradicular teeth excluding degrees of curvatures and wide canals ${ }^{17,18}$.

According to the results of the test in this study, the AH-Plus sealer showed significantly higher results than the MTA filapex and the Gutta flow, which came in accordance with a previous study that states that the AH-plus has unrivaled bond strength due to its low shrinkage, accompanied by the long term dimensional stability, ${ }^{19}$ in addition to the high penetration power into the micro irregularities which is a result of the creep capacity with a long setting time of this sealer. This in turn increases the mechanical interlock with the root canal radicular dentine, increasing its resistance to dislodgment and seal breakage ${ }^{20,21,22}$.

The low results of the MTA filapex sealer is most probably due to its inherent property that is releasing calcium and hydroxyl ions from the set sealer. These ions form appetite as they come in contact with phosphate containing fluids. Apetites then deposited in collagen fibrils and promote mineral nucleation forming tag like structures which have low adhesion properties $^{23}$

Gutta flow2 showed the lowest results which is in consistence with other studies ${ }^{24}$, this may be due to its poor wettability to dentine and the lack of leak-free seal between gutta-percha particles and the remainder of the sealer and the absence of chemical union between the master cone and the sealer material ${ }^{25}$

Gutta flow 2 is also characterized by poor wetting, which in turn prevents the sealer from entering the micro irregularities this is due to the presence of silicone particles which gives high surface tension leading to lower spread.

\section{CONCLUSIONS}

Within the limits of this experimental study, it can be concluded that the composition of endodontic sealers affects their push out bond strength, where the higher push out bond strength was found in GP/ AH Plus group, this result challenges the claim of the ongoing development in endodontic sealers that the conventional non bonding root canal filling era has come to an end, regardless of the theoretical concept behind the use of new novelties in endodontic sealers, continuous research and modifications are required to substitute what remains the simplest, cost effective and more reliable choice. 


\section{REFERENCES}

1. Ingle JI, Simon JH and Machtou BP. Outcome of endodontic treatment and re-treatment. In: Endodontics. BC Decker Inc 2002; 747-768.

2. Garrido ADB, Lia RCC, Franca SC, Da Silva JF, AstolfiFilho S, and Sousa-Neto MD. Laboratory evaluation of the physicochemical properties of a new root canal sealer based on Copaifera multijuga oil-resin. International Endodontic Journal 2010; 43(4):283-291.

3. Versiani MA, Carvalho-Junior JR, Padilha MIAF, Lacey S, Pascon EA, Sousa-Neto MD. A comparative study of physicochemical properties of AH Plus and Epiphany root canal sealants. Int Endod J 2006; 39:464-471.

4. Elayouti A, Achleithner C, Löst C, Weiger R. Homogenicity and adaptation of a new gutta-percha paste to root canal walls. J Endod. 2005; 31(9):687-90.

5. Kumar D, Shivanna V, Joshi V. Evaluation of guttaflow and guttapercha in filling of lateral grooves and depressions in a single rooted tooth - An in vitro study. Endodontology 2012; 24:35-41.

6. De-Deus G, Branda MC, Fidel RAS, Fidel SR. The sealing ability of GuttaFlow in oval-shaped canals: an ex vivo study using a polymicrobial leakage model. Int Endod J 2007; 40:794-799.

7. Torabinejad M, Parirokh M. Mineral trioxide aggregate: A comprehensive literature review-part II: leakage and biocompatibility investigations. J Endod 2010; 36:190-202.

8. Silva EJ, Rosa TP, Herrera DR. Evaluation of cytotoxicity and physicochemical properties of calcium silicate-based endodontic sealer MTA Fillapex. J Endod 2013; 39:274-7.

9. Panituisai P and Messer HH: Cuspal deflection in molars in relation to endodontic and restorative procedures. J Endod 1995; 21 2: 57-61.

10. Tagger M, Tagger E, Tjan AH and bakland LK: measurement of adhesion of endodontic sealers to dentine. J Endod. 2002; 28; 5: 351-4.

11. Huffman BP, Mai S, Pinna L, Weller RN, Primus CM, Gutmann JL: dislocation resistance of proroot sealers a calcium silicate based root canal sealer, from radicular dentine. IntEndod J. 2010; 43 :889-901

12. Goracci C, Tauares AU, Fabianelli A, Monticelli F, Raffaelli O, Cardoso PC. The adhesion between fiber post and root canal walls: comparison between micro tensile and push out bond strength measurement. Eur.J oral sci. 2004, 112(4):353-61.
13. Soares CJ, Santana FR, Castro CE, Santos PC, Soares PV, Qian F: finite element analysis and bond strength of glass post to interradicular dentine: comparison between micro tensile and pushout bond test. Dent Mater. 2008; 24 (10):1405-11.

14. Sadek FT, Monticelli F, Goracci C, Tay FR, Cordosa PE and Ferrari M: bond strength performance of different resin composites used as core materials around fiber posts. Dent mater.2007; 23 (1): 95-9.

15. Tyagi S, Mishra $\mathrm{P}$ and Tyagi $\mathrm{P}$. Evolution of root canal sealer. Euro J gen dent. 2013; 2: 199-218.

16. Gomes-filto JE, Watanabe S, Berhabe PF and De-Moraes Costa. MTA sealer stimulated mineralization J Endod 2009; 35: 256-60.

17. Gulabivala $\mathrm{K}$, Holt $\mathrm{R}$ and Long $\mathrm{B}$ an in vitro comparison of thermo placticized gutta percha obturation techniques with cold lateral condensation. Endod Dent Traumatol. 1998; 14: 262-9.

18. Zmener O and Banegas G. Clinical experience of root conduct fillinig by ultrasonic condensation of gutta-percha. Endod Dent Traumatol. 1999; 15: 57-9.

19. Nagas E, Uyanik M, Eymirli A: dentine moisture conditions affect the adhesion of root canal sealers. J Endod 2012; 38: 240-4.

20. Goiotti FA, Kuga MC, Duarte MA, S Antanna A, Junior and Faria E: Effect of calcium hydroxide dressing on pushout bond strength of endodontic sealers to root canal dentine. Braz Oral Res; 2014: 28.

21. Pommel L, About I, Pashley D, Camps J: Apical leakage of four endodontic sealers J Endod 2003; 29 (3): 208-10.

22. Numes VH, Silva RG, Alfredo E, Sousa-Neto MD and Silva Sousa YT: adhesion of epiphany and ah-plus sealers to human root dentine treated with different solutions. Braz Dent J. 2008; 19 (1): 46-50.

23. Sagsen B, Ustun Y, Demirbuger S and Pala K: push out bond strength of two new calcium silicate based endodontic sealers to root canal dentin. Int. Endod J.2011: 44; 1088-91.

24. Brackett ME, Martin R, Sword J, Martin RL, Schuster GS, Weller RN, Ferari M. Comparison of seal after obturation techniques using polymethyl siloxane based root canal sealers. J Endod 2006; 32: 1188-90.

25. Monticelli F, Sword J, Martin RL, Schuster GS, weller RN, Ferrari M. Sealing properties of two contemporary single cone obturation systems. Int Endod J. 2007; 40:374-8. 